\title{
Activity in the Circumstellar Envelope of the Be/Shell Star $\zeta$ Tau
}

\author{
Yulian Guo Lin Huang Jinxin Hao \\ Beijing Astronomical Observatory, Chinese Academy of Sciences
}

\section{Introduction}

$\zeta$ Tau is a well-known V/R-variable shell star. It is a single-lined spectroscopic binary with an orbital period of 133 days (Harmanec 1984, and Jarad 1987). Delplace (1970) found that the long-term radial velocity variations of the Balmer shell absorption lines are cyclic in 1960-67. Subsequently, similar behaviour has been observed and studied by several authors (Delplace and Chambon 1976, Hubert-Delplace et al 1983, Harmanec 1984, and Guo and Cao 1987). Mon et al (1992) showed that the cyclic variation had terminated and the star seems to have entered a new quiet phase around 1982.

\section{Observations and Data Reduction}

All observations were carried out at Xinglong station of Beijing Observatory. The spectroscopic data were obtained using the grating spectrograph attached to the $60 / 90 \mathrm{~cm}$ Schmidt telescope with plate during 1978 Dec.-1990 Feb.. After 1990 Dec., spectroscopic observations were made with the All-Fiber-Coupler grating spectrograph at the $216 \mathrm{~cm}$ telescope with a CCD detector with $576 \times 384$ pixels. The reciprocal liner dispersions of the spectrum were $17 \AA / \mathrm{mm}, 50 \AA / \mathrm{mm}$ and $86 \AA / \mathrm{mm}$ at the blue region and $50 \AA / \mathrm{mm}$ and $86 \AA / \mathrm{mm}$ at the $\mathrm{H}_{\alpha}$ region. Spectrograms have been digitized on the PDS microdensitometer of the Purple Mountain Observatory. All spectroscopic data are reduced with Starlink image processing software on the VAX 11/780 computer of Beijing Observatory. To analyse and study the shell activity, we measured the radial velocities of the stronger shell lines. The photometry was made on the $60 \mathrm{~cm}$ reflector using the filters of the standard UBV system during 1984 Dec.-1992 Dec.

\section{Results and Discussion}

Fig.1a shows the variations of the mean velocity of $\mathrm{H}_{\gamma}, \mathrm{H}_{\delta}$ and $\mathrm{H}_{\epsilon}$ shell lines with time. It seems that the cyclic variations which started in 1976 (Huber-Delplace et al. 1983) ceased around 1982. The velocity values still displayed an oscillation of the cycle in 1982-1985 but the cycle lasted shorter and the amlitude was less. During 1986-1990, the variations of the radial velocity conspicuously diminished and the cyclic variation disappeared too.

The variations of the $V / R$ ratio of $\mathrm{H}_{\alpha}$ is in phase with the radial velocity curve of the shell absorption lines before 1982. Between 1983 and 1987, the $\mathrm{V} / \mathrm{R}$ ratios fluctuated in an irregular manner but remained generally close to unity. After 1987 the cyclic changes of the $V / R$ ratio reappeared.(see Fig.1b).

The equivalent width of $\mathrm{H}_{\alpha}$ emission declined gradually from 1978 to 1988 , but afterwards, it began to rise rapidly, although there could be some fluctuations in-between.(see Fig.1c).

In addition to the above-mentioned long-term variations, we also observed some short-term activities on a time scale of night-to-night and even more rapid changes in $\mathrm{H}_{\alpha}$ line. For example, the $\mathrm{H}_{\alpha}$ profiles obtained on 1983 Dec. 10-14, appeared remarkable variations on line shape. The equivalent widths of the $\mathrm{H}_{\alpha}$ emission increased from 10.0 Åto 15.0Ån a 4-night interval.

during 1990-1992, the brightness of the star showed evident variation which was correlated to the $V / R$ variation of $H_{\alpha}$ and the minimum of the brighness occurred between the end of 1991 and the beginning of 1992. In addition the rapid change of the UBV magitudes were reveated in 1984 Dec.-1985 Jan. and the beginning of 1989(see Fig.1d, e and f). 
Our spectroscopic observations indicate that the $V / R$ cyclic variations of $H_{\alpha}$ emission emerged once again at the end of 1990 after their disappearance for several years. We do not have the necessary information about the radial velocity after 1990 but, based on the correlation between the $\mathrm{V} / \mathrm{R}$ and the radial velocity variation revealed in our observations in 1978-1982 and those of Hubert-Delplace et al. (1982) in $1960-1981$, we may suggest that the radial velocity could have entered new cyclic variation phase. To our best knowledge, there are two possible interpretations to the origin of the $V / R$ cycle associated with the radial velocity curves: the rotating-pulsating envelope and the non-axisymmetric elliptical disk. We consider that an elliptical disk model in slow precession motion appears more convincing for explaining the various variations we observed in the cyclic variation phase. But other observed facts, such as that the periods and the amplitudes from one cycle to another were different and that the cyclic variations disappeared completely as in 19851987 , can't be interpreted only in terms of a simple slowly rotating elliptical envelope. In modelling the envelope of the star we need to consider the stage which is without the presence of the cyclic change, namely, usually what is called a quiet stage and the process of the disappearance of the cyclic change. In addition, we observed important short-term variations. The rapid changes can be due to erratic activities of matter streams inside the envelope. Affected by such matter stream activities, the elliptical disk will vary with time. A sufficiently strong activity could even have destroyed elliptical disk. In consequence,the cyclic variation vanished. Of course owing to other violent activities of matter and accumulations of the outflow matter, a new elliptical disk could form again. In the meantime,the cyclic variations are observed. Therefore we consider that a model which best explains the behaviour of the envelope of $\zeta$ Tau, would be represented by the coexistence of and interaction between an elliptical disk in slow precessional motion and highly variable streams of matter.

\section{Reference:}

1. Delplace, A. M. 1970 , A. Ap. 7,68

2. Delplace, A. M. and Chambon, M. Th., 1976, in IAU symp. No.70, Be and shell stars ed A. Slettebak (Dordrecht:Reide), P.79

3. Guo Yulian and Gao Weishi, 1987, Be Newsletter 16,9

4. Harmanec, P., 1984, Bull. Astron. Inst. Czech. 35, 164

5. Hubert-Delplace, A. M., Jaschek, M., Hubert, H. and Chanbon, M. Th.,1982, in IAU symp. 98, eds. M. Jaschek and M. G. Groth, P.125

6. Hubert-Delplace, A. M., Mon. M., Ungerer, V., Hirata, R., Paterson- Beeckmans, F., Hubert, H., and Baade,D., 1983, A. Ap. 121, 174

7. Jarad, M. M., 1987, Ap. S. S. 139,83

8. Mon, M., Kogure, T., Suzuki, M., and

Singh, M., 1992, Publ. Astron. Soc. Japan,44,73

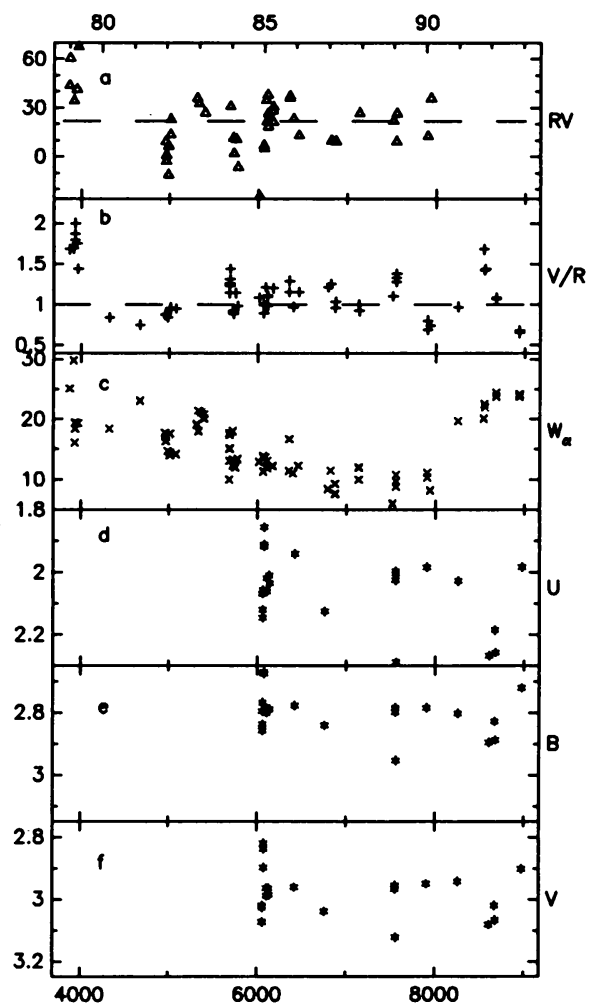

